\title{
Potential Drug Target Identification of Legionella pneumophila by Subtractive Genome Analysis: An In Silico Approach
}

\author{
Md. Sadikur Rahman Shuvo1, Shahriar Kabir Shakil ${ }^{2}$, Firoz Ahmed ${ }^{1 *}$ \\ ${ }^{1}$ Department of Microbiology, Noakhali Science and Technology University, Noakhali-3814, Bangladesh. \\ ${ }^{2}$ Department of Biotechnology and Genetic Engineering, Noakhali Science and Technology University, Noakhali-3814, Bangladesh.
}

\begin{abstract}
Though Legionella pneumophila is an opportunistic pathogen, recent reports about multi drug resistance in $L$. pneumophila is alarming. Annotated whole genome provides a pool of information which is applied for therapeutic drug targets identification in pathogenic bacteria. Subtractive genomic analysis is a pragmatic approach to screen the essential proteins present in pathogen but absent in host. Phylogenetically closely related L. pneumophila str. Philadelphia and $L$. pneumophila str. ATCC43209 protein profiles were analyzed to identify putative drug targets. Paralogous duplicate profiles were primarily discarded using CD-hit suit. Six hundred and ninety one L. pneumophila str. Philadelphia and 690 L. pneumophila str. ATCC43209 human homologous proteins were excluded using blstP. Among the human non-homologous proteins, the essential proteins for bacteria were separated using DEG tool. For both strains, one hundred and nineteen essential proteins were marked which participate in various metabolic pathways. Among them 11 unique proteins were found. Beside it, 15 and 16 exposed surface proteins were present in strain Philadelphia and ATCC43209 respectively. These unique and cell surface proteins can be utilized for effective drug and vaccine targets.
\end{abstract}

Keywords: Phylogeny, Essential genes, Drug target, Unique pathway

\section{Introduction}

The Legionellaceae are Gram-negative bacteria found in aquatic environments all over the world. Usually they are intracellular parasites of free-living protozoa. They are also distributed in manmade water systems where they survived freely in biofilms. The family Legionellaceae consists of a single genus, Legionella. More specifically, this genus includes the species L. pneumophila, which are non-encapsulated, aerobic bacilli. L. pneumophila is an opportunistic pathogen that causes infections in immunocompromised individuals. The bacterium is most notable as the causative agent of Legionnaires' disease, a potentially fatal pneumonia ${ }^{1}$.

This organism was identified in 1976 in Philadelphia when an outbreak of a serious pneumonia occurred in individuals attending an annual convention. Approximately 200 people developed pneumonia within the first few days after the convention and about 2 dozen succumbed to respiratory failure ${ }^{2}$. This outbreak of pneumonia in the hotel was initially suspected to cause by toxic substances or some other environmental problems.

After rigorous investigations from Centers for Disease Control and Prevention (CDC), a unique bacterium was identified several months later and thought to be a new microbe ${ }^{3-4}$. The organism was named Legionella because the disease was identified first in those attending the Legionnaire's convention. Some previous outbreaks of pneumonia had become evident caused few decades back ${ }^{5}$.
By subculturing into a rich artificial medium, L. pneumophila, was first isolated by inoculation of postmortem lung tissue into guinea pigs ${ }^{4}$. By indirect immunofluorescent antibody assay, it was discovered that a number of unexplained respiratory disease were associated with seroconversion to L. pneumophila, a "rickettsia-like" organism, isolated by guinea pig inoculation from the blood of a feverish patient in 1947, which today is recorded as the earliest known isolate of L. pneumophila ${ }^{6}$.

In the moderately brief time frame, L. pneumophila was first distinguished as a human pathogen, in excess of 50 types of Legionella have been perceived, and no less than 24 of these have been related with human disease. It is conceivable that under the fitting conditions, immunocompromised individuals can be tainted with any types of Legionella. The incredible larger part of Legionnaires' ailment, around $90 \%$, is caused by $L$. pneumophila, and notwithstanding the portrayal of somewhere around 15 serogroups, L. pneumophila serogroup 1 is in charge of over $84 \%$ of cases around the world ${ }^{7-9}$.

It has been accounted for that L. pneumophila is getting to be impervious to specific anti-infection agents, for example, erythromycin, rifampicin and quinolones derivatives ${ }^{10-13}$. The development of medication obstruction of L. pneumophila has prompted the look for novel medication targets. The accessibility of finish genome successions of L. pneumophila strain Philadelphia has cleared the better approach to recognize the 
novel medication targets. The goal of present work was to distinguish putative medication focuses in L. pneumophila strain Philadelphia through metabolic pathway examination, to play out the homology displaying and to play out the atomic elements reenactment of a candidate drug target.

\section{Materials and Methods:}

Sequences of methodology are summarized in Figure 1.

Phylogenetic analysis:

Phylogenetic analysis of L. pneumophila str. Philadelphia (Accession: NC_002942) and L. pneumophila str. ATCC43209 (Accession: NC_016811) was performed by PanX phylogeny ${ }^{14}$ against 85 other $\bar{L}$. pneumophila sequences available in National Center for Biotechnology Information (NCBI). Gene parameters of these two strains were analyzed.

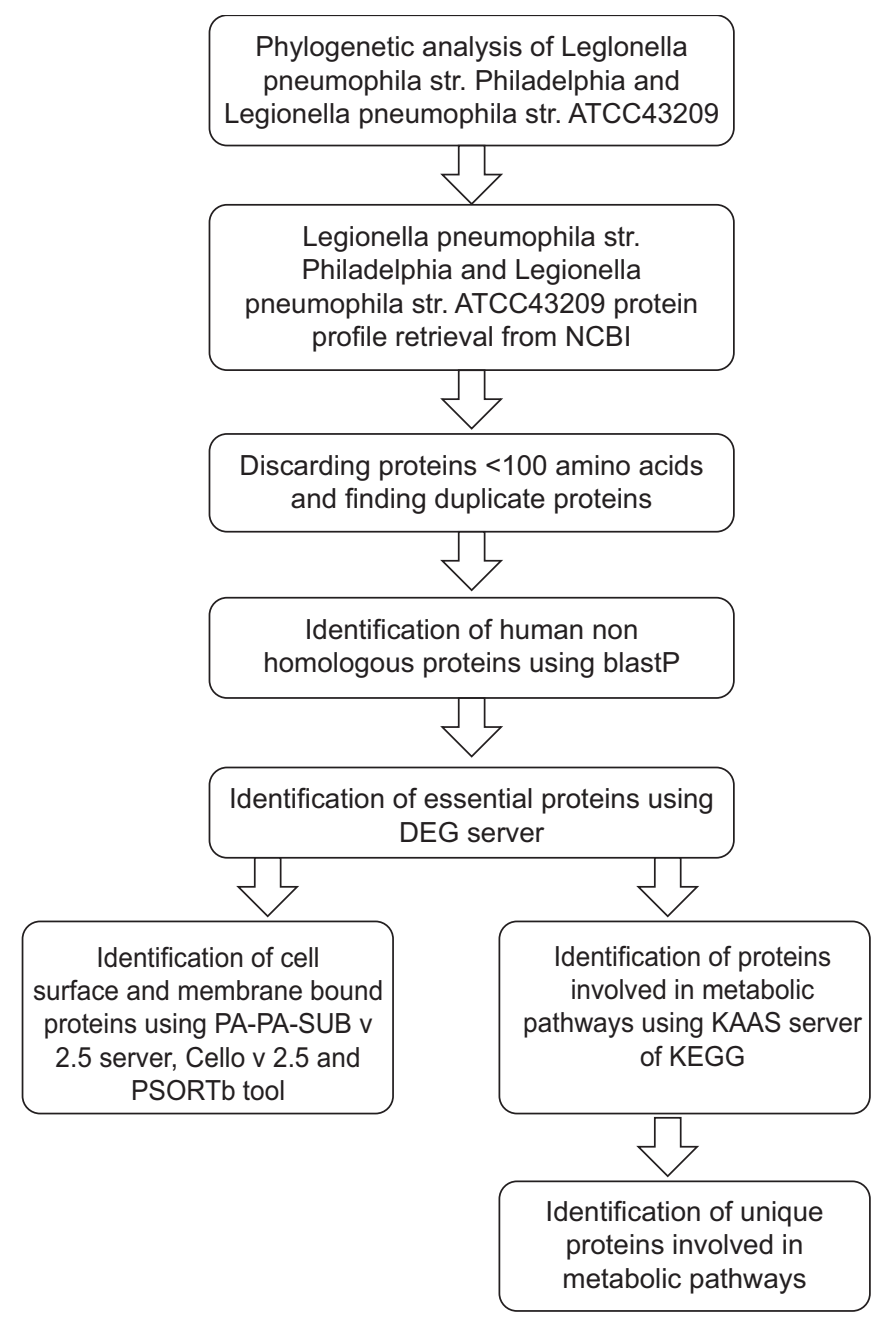

Figure 1. Schemetic diagram of methodology.

\section{Retrieval of protein sequences:}

Complete information of L. pneumophila str. Philadelphia (Accession: NC_002942) and L. pneumophila str. ATCC43209 (Accession: NC_016811) was retrieved from National Center for Biotechnology Information (https://www.ncbi.nlm.nih.gov/) as GenBank file format. All protein sequences were retrieved from the GenBank files using Bio-Python ${ }^{15}$ module and saved as fasta file format for the following assays. Fasta comments were trimmed from the multiple fasta files in such a way that they contain only the accession number of respective protein sequences for efficient tagging and easy recognition.

\section{Paralog proteins identification:}

The duplicate protein sequences of $L$. pneumophila str. Philadelphia and str. ATCC43209 were removed from the master fasta files. The multiple fasta files were subjected to CD-hit suit web server ${ }^{16}$ which uses sequence identity at cut off value $0.60 \%{ }^{17}$. After the duplicate proteins were separated from the master fasta file, it only contained non-paralog proteins. The sequences were then screened to have at least 100 amino acids in their chains. The protein sequences which had less than 100 amino acids in their chains were excluded from the master database.

\section{Human non homolog protein identification:}

The non paralog proteins found in the previous stage were then subjected to NCBI blastP against Homo sapiens. The purpose was to find out the proteins those are homolog to the human genome. At this stage the threshold expectation value was 0.0001 . The proteins those showed similarity at this threshold value were removed from the database. The rest of the proteins remained in the database are human non-homologous proteins which will be ultimately used for the identification of drug target in the subsequent stages.

Search for essential proteins in the database:

Database of essential genes (DEG) ${ }^{18}$, an web server, providing the facility of essential protein identification, was used to find the essential genes in L. pneumophila str. Philadelphia and str. ATCC43209. In this case maximum threshold value was $10^{-100}$ and bit score cut off value was 100 . Output from this analysis gives the essential human non-homologous essential proteins of L. pneumophila str. Philadelphia and str. ATCC43209. The essential proteins were then categorized based on the metabolic activity by blastKoala ${ }^{19}$.

Metabolic pathway analysis of essential proteins:

KEGG Automatic Annotation Server KASS ${ }^{20}$ (https:// www.genome.jp/tools/kaas/) was used for the analysis of metabolic pathways of essential proteins. This is extremely helpful to identify the unique targets. In this analysis functional annotation of genes are performed where manually arranged KEGG gene database is used for template search. The output result comprises of KEGG Ontology assignments and KEGG pathways.

Unique pathway analysis:

KEGG Genome Database was used for the identification of unique metabolic pathway prevailing in L. pneumophila str. Philadelphia and str. ATCC43209. The metabolic pathways of Homo sapiens and the two pathogenic strains of $L$. pneumophila were compared. The pathway map generated in this step 
represents the unique metabolic machineries in L. pneumophila str. Philadelphia and str. ATCC43209.

Identification of cell surface proteins:

Function of a protein can easily be portended if location of it is predicted. Bacterial surface proteins are always good objective for drug and vaccine.

PA-SUB server ${ }^{21}$ (Proteome Analyst Specialized Subcellular Localization Server v2.5) (http://webdocs.cs.ualberta.ca/ $\sim$ bioinfo/PA/Sub/) was used for the prediction of particular localization of the necessary proteins identified in the previous stages. Results were verified by doing the same analysis in two other web tools, PSORTb ${ }^{22}$ and CELLO v2. $5^{23}$

\section{Result and Discussion:}

Prime purpose of this study is to look into the effective drug target by systematic subtraction of genes from genome. In this study we worked with two different strains of L. pneumophila. At first their evolutionary relationship was verified with other 85 strains of L. pneumophila by whole genome phylogenetic analysis. The phylogenetic study (Figure 2) shows that the two strains considered in this study have high similarities with most of the other L. pneumophila strains. Through in-silico analysis, such genes were identified those are absent in host Homo sapiens. The result of subtractive analytical steps is summarized in Table 1.

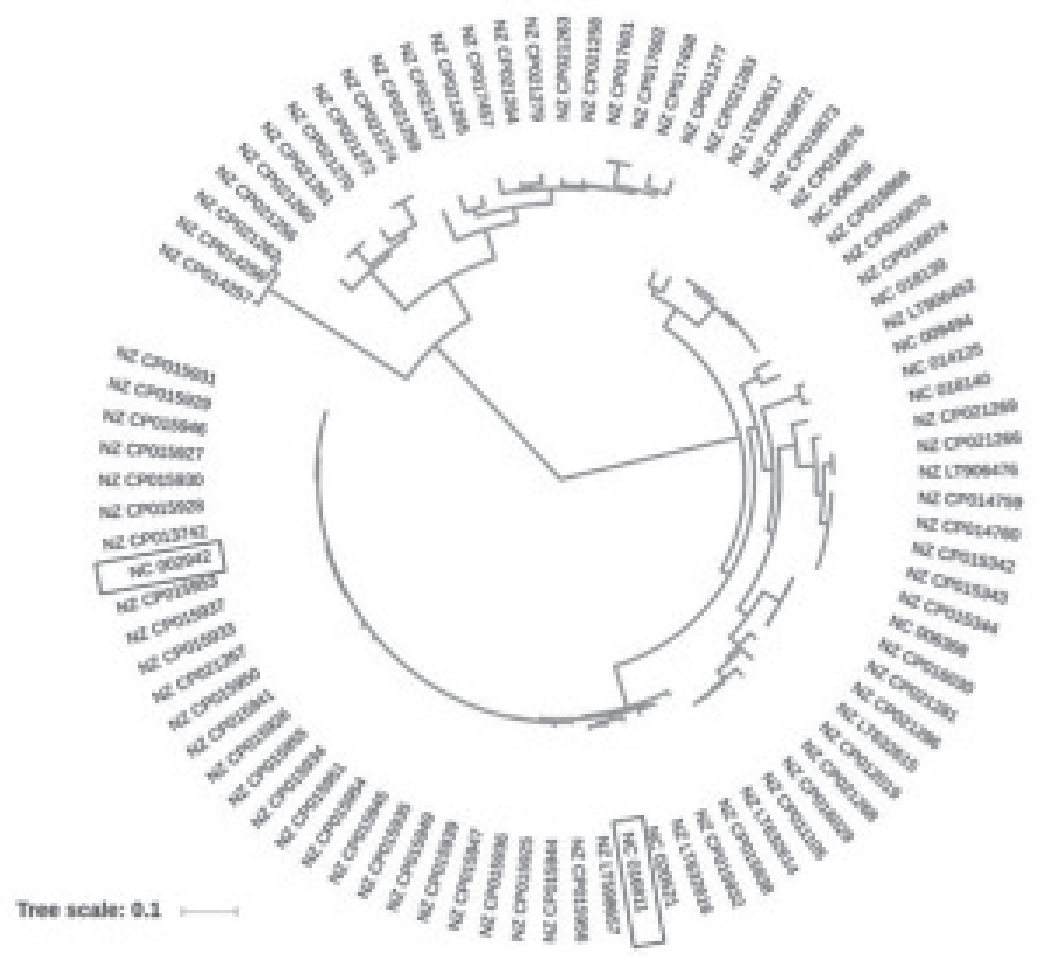

Figure 2. Whole genome phylogenetic analysis of Legionella pneumophila str. Philadelphia (Accession: NC_002942) and Legionella pneumophila str. ATCC43209 (Accession: NC_016811) against other 85 strains of Legionella pneumophila.

Table 1. Subtractive analytical output

\begin{tabular}{lcc}
\hline Analytical steps & \multicolumn{2}{c}{ Total number of proteins } \\
\cline { 2 - 3 } & $\begin{array}{c}\text { Legionella pneumophila } \\
\text { str. Philadelphia }\end{array}$ & $\begin{array}{c}\text { Legionella pneumophila } \\
\text { str. ATCC43209 }\end{array}$ \\
\hline Retrieved protein & 2931 & 3020 \\
Proteins $>100$ amino acids & 2744 & 2774 \\
Non-paralogous proteins & 2698 & 2702 \\
Human homologous proteins & 691 & 690 \\
Human non-homologous proteins & 2007 & 2012 \\
Essential proteins predicted by DEG & 301 & 302 \\
Essential proteins in metabolic pathway & 119 & 119 \\
Cell surface essential proteins & 15 & 16 \\
Proteins involved in unique pathways & 11 & 11 \\
\hline
\end{tabular}


A total of 2931 L. pneumophila str. Philadelphia and $3020 \mathrm{~L}$. pneumophila str. ATCC43209 protein sequences were retrieved. Less than 100aa sequence length proteins were excluded. After exclusion 2744 L. pneumophila str. Philadelphia and $2774 \mathrm{~L}$. pneumophila str. ATCC43209 proteins were used for next step. After filtering in CD hit, 2698 L. pneumophila str. Philadelphia and 2702 L. pneumophila str. ATCC43209 remained. Threshold value for CD-hit was $60 \%$. The blast search result in NCBI showed $691 \mathrm{~L}$. pneumophila str. Philadelphia and $690 \mathrm{~L}$. pneumophila str. ATCC43209 proteins which are human homologous. Ultimately 2007 L. pneumophila str. Philadelphia and 2012 L. pneumophila str. ATCC43209 proteins identified which are human non homologs. Evolutionary consequences dictate the sharing of some common genes in host and bacteria. Even they are involved in similar cellular systems. In this case proteins having insignificant similarity were considered as nonhomologous proteins.

Database of Essential Genes (DEG) screened 302 L. pneumophila str. Philadelphia and 301 L. pneumophila str. ATCC43209 proteins which are essential proteins in pathogen. BlastKoala categorized the essential proteins according to their functions (Figure 3). All these proteins are human non-homologous and at the same time they have key role in cellular activities. These proteins were again subjected to KAAS server at KEGG to find which metabolic pathways they are involved. KAAS is a BLAST based analysis tool which analyzes prokaryotic proteome against host proteome. KAAS analysis gave an output of 119 essential proteins which are directly involved in metabolic pathways. This is a vital stage of screening, because the proteins found in this step are involved in major metabolic activities of bacteria. So, targeting these proteins we can design drug which can deactivate one or more metabolic pathways and making the bacteria susceptible to that drug.

By the comparative analysis of the metabolic pathway of host and the pathogen (L. pneumophila) in KEGG, both $L$. pneumophila str. Philadelphia and L. pneumophila str. ATCC43209 showed five uncommon metabolic pathways have been found which are not present in human. Eleven proteins of L. pneumophila str. Philadelphia are involved in these pathways which can be used for drug target Table 2. Same proteins were found in L. pneumophila str. ATCC43209 while performed BLAST search against $L$. pneumophila str. Philadelphia. These proteins play vital role for survival and regulatory mechanism in
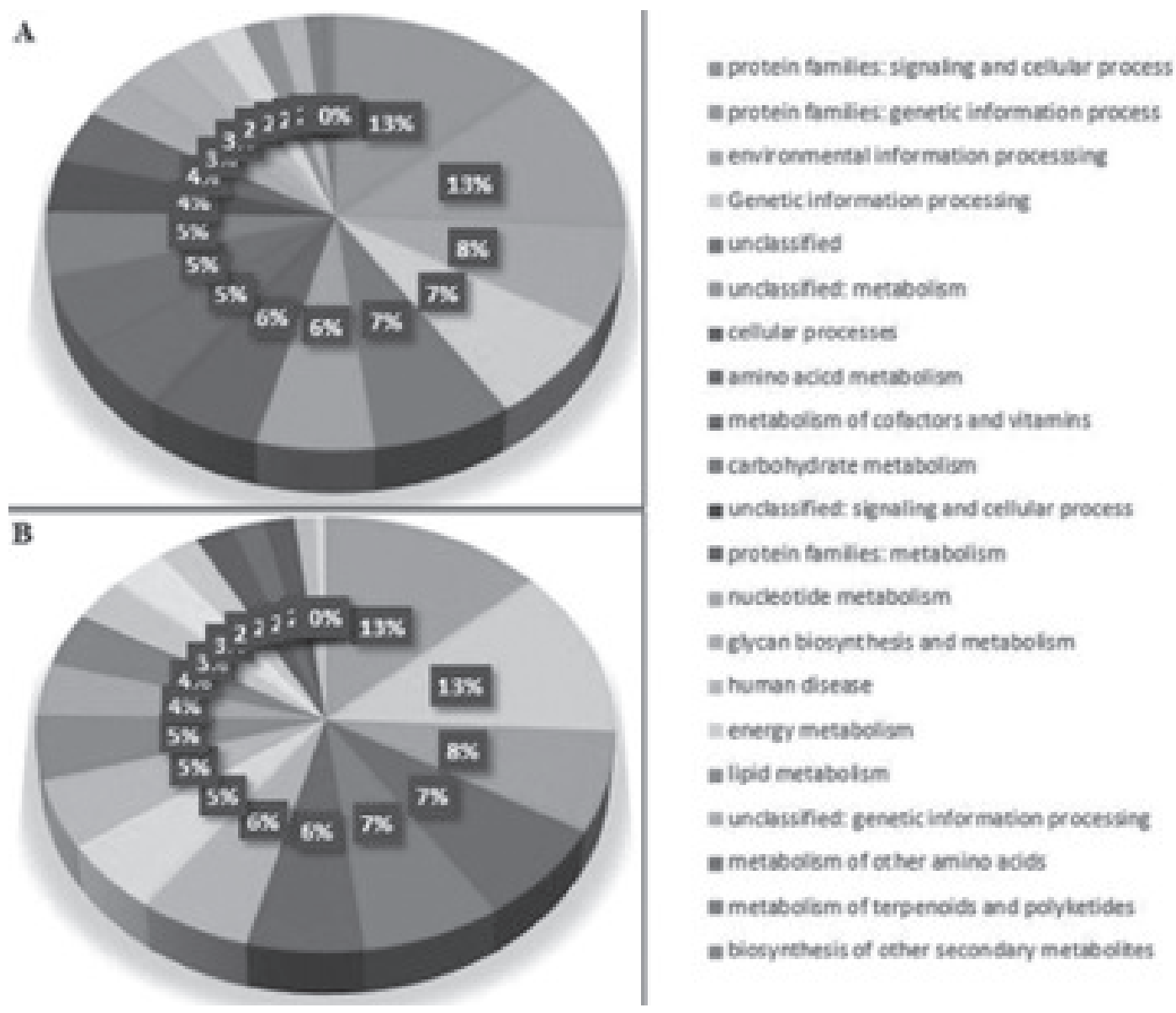

Figure 3. Categories of essential proteins based on their functions in Legionella pneumophila str. Philadelphia (A) and Legionella pneumophila str. ATCC43209 (B). 
bacteria. Thus these proteins must have high possibility of being good drug target.

Prediction of the position of proteins in bacteria was done by PA-SUB v 2.5 server, CELLO v2.5 and PSORTb server. Those proteins which showed cell surface localization by the entire three prediction tool were taken consideration as cell surface protein. List of the proteins as well as their function is listed in Table 3.
Both strains of L. pneumophila showed same type of cell surface protein. The hypothetical protein's function was predicted using SVMProt web server ${ }^{24}$ based on P value. Among the hypothetical proteins, 2 are zinc binding protein and 2 are lipid binding protein. One of the lipid binding proteins is putative transport protein. All these proteins can be good target for vaccine design to control disease caused by L. pneumophila.

Table 2. List of proteins involved in unique pathways in Legionella pneumophila.

\begin{tabular}{ll}
\hline $\begin{array}{l}\text { Protein accession numberof Legionella } \\
\text { pneumophila } \text { str. Philadelphia }\end{array}$ & Protein name \\
\hline WP_010946303.1 & desC; stearoyl-CoA desaturase \\
WP_010948619.1 & transcription termination factor \\
WP_010946024.1 & MFS transporter, UMF1 family \\
WP_010948309.1 & ftsZ; cell division protein FtsZ \\
WP_010947297.1 & hydroxymethylpyrimidine kinase \\
WP_010946377.1 & ATP-dependent HslUV protease \\
WP_010946112.1 & htrB; Kdo2-lipid IVA lauroyltransferase \\
WP_010948046.1 & 3-deoxy-D-manno-octulosonic-acid transferase \\
WP_010946259.1 & UDP-N-acetylglucosamine acyltransferase \\
WP_010947281.1 & UDP-2,3-diacylglucosamine hydrolase \\
WP_010946499.1 & Fuc2NAc and GlcNAc transferase
\end{tabular}

Table 3. List of cell surface proteins and their functions.

\begin{tabular}{|c|c|c|c|c|c|}
\hline $\begin{array}{l}\text { Legionella pneumophila } \\
\text { str. Philadelphia }\end{array}$ & Protein name & $\mathrm{P}$ value & $\begin{array}{c}\text { Legionella pneumophila } \\
\text { str. ATCC43209 }\end{array}$ & Protein name & $P$ value \\
\hline WP_011213753.1 & $\begin{array}{l}\text { Hypothetical protein } \\
\text { (lipid binding protein) }\end{array}$ & 0.90 & WP_010946366.1 & $\begin{array}{l}\text { Pilus assembly } \\
\text { protein }\end{array}$ & 0.99 \\
\hline WP_011945791.1 & $\begin{array}{l}\text { Pilus assembly } \\
\text { protein }\end{array}$ & 0.99 & WP_010946601.1 & $\begin{array}{l}\text { DUF4156 domain } \\
\text { containing protein }\end{array}$ & 0.99 \\
\hline WP_014326896.1 & $\begin{array}{l}\text { Thaumatin domain } \\
\text { containing protein }\end{array}$ & 0.99 & WP_010948117.1 & $\begin{array}{l}\text { Hypothetical protein } \\
\text { (lipid binding protein) }\end{array}$ & 0.91 \\
\hline WP_010948117.1 & $\begin{array}{l}\text { Hypothetical protein } \\
\text { (Putative transport protein) }\end{array}$ & 0.91 & WP_010946952.1 & $\begin{array}{l}\text { Flagellar basal body } \\
\text { rod protein FlgF }\end{array}$ & 0.99 \\
\hline WP_010946953.1 & $\begin{array}{l}\text { Flagellar basal body } \\
\text { rod protein FlgG }\end{array}$ & 0.99 & WP_010946896.1 & Membrane protein & 0.99 \\
\hline WP_010946952.1 & $\begin{array}{l}\text { Flagellar basal body } \\
\text { rod protein FlgF }\end{array}$ & 0.99 & WP_010946948.1 & $\begin{array}{l}\text { Flagellar basal body } \\
\text { rod protein FlgB }\end{array}$ & 0.99 \\
\hline WP_010947070.1 & Flagellin & 0.99 & WP_010947197.1 & $\begin{array}{l}\text { Hypothetical protein } \\
\text { (lipid binding protein) }\end{array}$ & 0.91 \\
\hline WP_010945781.1 & $\begin{array}{l}\text { Peptidase M4 family } \\
\text { protein }\end{array}$ & 0.99 & WP_010947059.1 & $\begin{array}{l}\text { Thaumatin domain } \\
\text { containing protein }\end{array}$ & 0.99 \\
\hline WP_010946850.1 & $\begin{array}{l}\text { Hypothetical protein } \\
\text { (Zinc binding protein) }\end{array}$ & 0.92 & WP_010946850.1 & $\begin{array}{l}\text { Hypothetical protein } \\
\text { (Zinc binding protein) }\end{array}$ & 0.92 \\
\hline WP_010947068.1 & $\begin{array}{l}\text { Flagellar hook } \\
\text { protein FID }\end{array}$ & 0.99 & WP_010947068.1 & $\begin{array}{l}\text { Flagellar hook } \\
\text { protein FID }\end{array}$ & 0.99 \\
\hline WP_010948122.1 & Penicillin binding protein & 0.99 & WP_010948122.1 & Penicillin binding protein & 0.99 \\
\hline WP_014326669.1 & $\begin{array}{l}\text { Type I secretion C- } \\
\text { terminal target } \\
\text { domain containing protein }\end{array}$ & 0.99 & WP_010946382.1 & $\begin{array}{l}\text { Type I secretion C- } \\
\text { terminal target } \\
\text { domain containing protein }\end{array}$ & 0.99 \\
\hline
\end{tabular}


In this study a subtractive manner was applied for identification of drug target in L. pneumophila. The same approach has been applied for several other pathogenic microorganisms ${ }^{25,26,27}$. This will be helpful for drug development in the further studies.

\section{Conclusion}

In our study, we involved two different strains of L. pneumophila. Relatedness of the two strains were verified by phylogenetic analysis. Subtractive genome analysis finally found 11 unique proteins in both strains which are involved in unique metabolic pathways of L. pneumophila. These proteins are non-homologous to human genome. The unique proteins can be analyzed by laboratory experimental analysis for drug target in future. We also found 15 and 16 cell surface proteins in Philadelphia and ATCC43209 respectively that will be useful for vaccine target identification.

\section{References}

1. Brenner DJ, Steigerwalt AG and McDADE JE. 1979. Classification of the Legionnaires' disease bacterium: Legionella pneumophila, genus novum, species nova, of the family Legionellaceae, familia nova. Ann Intern Med., 90(4): 656-658.

2. Newton HJ, Ang DK, van Driel IR and Hartland EL. 2010. Molecular pathogenesis of infections caused by Legionella pneumophila. Clin Microbiol Rev., 23(2): 274-298.

3. Fraser DW, Tsai TR, Orenstein W, Parkin WE, Beecham HJ, Sharrar RG, Harris J, Mallison GF, Martin SM, McDade JE and Shepard CC. 1977. Legionnaires' disease: description of an epidemic of pneumonia. New Engl J Med., 297(22): 1189-1197.

4. McDade JE, Shepard CC, Fraser DW, Tsai TR, Redus MA, Dowdle WR and Laboratory Investigation Team*. 1977. Legionnaires' disease: isolation of a bacterium and demonstration of its role in other respiratory disease. New Engl J Med., 297(22): 1197-1203.

5. Fang G, Yu VL and Vickers RM. 1989. Disease due to the Legionellaceae (other than Legionella pneumophila). Historical, microbiological, clinical, and epidemiological review. Medicine, 68(2): 116-132.

6. Hébert GA, Moss CW, McDougal LK, Bozeman FM, McKinney RM and Brenner DJ. 1980. The rickettsia-like organisms Tatlock (1943) and HEBA (1959): bacteria phenotypically similar to but genetically distinct from Legionella pneumophila and the WIGA bacterium. Ann Intern Med., 92(1): 45-52.

7. Marston BJ, Plouffe JF, File TM, Hackman BA, Salstrom SJ, Lipman HB, Kolczak MS and Breiman RF. 1997. Incidence of communityacquired pneumonia requiring hospitalization: results of a populationbased active surveillance study in Ohio. Arch Intern Med., 157(15): 17091718.

8. Muder RR and Victor LY. 2002. Infection due to Legionella species other than L. pneumophila. Clin Infect Dis., 35(8): 990-998.

9. Yu VL, Plouffe JF, Pastoris MC, Stout JE, Schousboe M, Widmer A, Summersgill J, File T, Heath CM, Paterson DL and Chereshsky A. 2002. Distribution of Legionella species and serogroups isolated by culture in patients with sporadic community-acquired legionellosis: an international collaborative survey. J Infect Dis., 186(1): 127-128.
10. Moffie BG and Mouton RP. 1988. Sensitivity and resistance of Legionella pneumophila to some antibiotics and combinations of antibiotics. $J$ Antimicrob Chemoth., 22(4): 457-462.

11. Walz A, Nichterlein T and Hof H. 1997. Excellent activity of newer quinolones on Legionella pneumophila in J774 macrophages. Zbl Bakt., 285(3): 431-439.

12. Tsakris A, Alexiou-Daniel S, Souliou E and Antoniadis A. 1999. In-vitro activity of antibiotics against Legionella pneumophila isolates from water systems. J Antimicrob Chemoth., 44(5): 693-695.

13. Fong DH, Lemke CT, Hwang J, Xiong B and Berghuis AM. 2010. Structure of the antibiotic resistance factor spectinomycin phosphotransferase from Legionella pheumophila. J Biol Chem., pp.jbcM109.

14. Ding W, Baumdicker F and Neher RA. 2017. panX: pan-genome analysis and exploration. Nucleic Acids Res., 46(1): e5-e5.

15. Cock PJ, Antao T, Chang JT, Chapman BA, Cox CJ, Dalke A, Friedberg I, Hamelryck T, Kauff F, Wilczynski B and De Hoon MJ. 2009. Biopython: freely available Python tools for computational molecular biology and bioinformatics. Bioinformatics, 25(11): 1422-1423.

16. Huang Y, Niu B, Gao Y, Fu L and Li W. 2010. CD-HIT Suite: a web server for clustering and comparing biological sequences. Bioinformatics, 26(5): 680-682.

17. Kumar GS, Sarita S, Kumar GM, Pant KK and Seth PK. 2010. Definition of potential targets in Mycoplasma Pneumoniae through subtractive genome analysis. J. Antivir. Antiretrovir, 2: 038-041.

18. Zhang R, Ou HY and Zhang CT, 2004. DEG: a database of essential genes. Nucleic Acids Res, 32(suppl_1): D271-D272.

19. Kanehisa M, Sato Y and Morishima K. 2016. BlastKOALA and GhostKOALA: KEGG tools for functional characterization of genome and metagenome sequences. $J$ Mol Biol., 428(4): 726-731.

20. Moriya Y, Itoh M, Okuda S, Yoshizawa AC and Kanehisa M. 2007. KAAS: an automatic genome annotation and pathway reconstruction server. Nucleic Acids Res., 35(suppl_2): W182-W185.

21. Lu Z, Szafron D, Greiner R, Lu P, Wishart DS, Poulin B, Anvik J, Macdonell $\mathrm{C}$ and Eisner R. 2004. Predicting subcellular localization of proteins using machine-learned classifiers. Bioinformatics, 20(4): 547-556.

22. Yu NY, Wagner JR, Laird MR, Melli G, Rey S, Lo R, Dao P, Sahinalp SC, Ester M, Foster LJ and Brinkman FS. 2010. PSORTb 3.0: improved protein subcellular localization prediction with refined localization subcategories and predictive capabilities for all prokaryotes. Bioinformatics, 26(13): 1608-1615.

23. Yu CS, Lin CJ and Hwang JK. 2004. Predicting subcellular localization of proteins for Gram negative bacteria by support vector machines based on peptide compositions. Protein Sci., 13(5): 1402-1406.

24. Cai CZ, Han LY, Ji ZL, Chen X and Chen YZ. 2003. SVM-Prot: web-based support vector machine software for functional classification of a protein from its primary sequence. Nucleic Acids Res., 31(13): 3692-3697.

25. Uddin R and Saeed K. 2014. Identification and characterization of potential drug targets by subtractive genome analyses of methicillin resistant Staphylococcus aureus. Comput Biol Chem., 48: 55-63.

26. Galperin MY and Koonin EV. 1999. Searching for drug targets in microbial genomes. Curr Opin Biotech., 10(6): 571-578.

27. Sakharkar KR, Sakharkar MK and Chow VT. 2004. A novel genomics approach for the identification of drug targets in pathogens, with special reference to Pseudomonas aeruginosa. In sili Biol., 4(3): 355-360. 